\title{
Derivation and validation of a risk-factor model for detection of oral potentially malignant disorders in populations with high prevalence
}

\author{
HK Amarasinghe ${ }^{*, 1,3}$, NW Johnson ${ }^{2,3}$, R Lalloo ${ }^{2,3}$, M Kumaraarachchi $^{3}$ and S Warnakulasuriya ${ }^{4}$ \\ 'Dental Institute, Maharagama, Colombo 10280, Sri Lanka; '2 Griffith Health Institute, Gold Coast Campus, Griffith University, Queensland 4222, \\ Australia; ${ }^{3}$ School of Dentistry and Oral Health, Gold Coast Campus, Griffith University, Queensland 4222, Australia; ${ }^{4}$ Department of Oral Medicine and \\ Experimental Oral Pathology, King's College Dental Institute, London SES 9RS, UK
}

\begin{abstract}
BACKGROUND: Oral and pharyngeal cancers constitute the sixth most common type of cancer globally, with high morbidity and mortality. In many countries, most cases of oral cancer arise from long-standing, pre-existing lesions, yet advanced malignancies prevail. A new approach to early detection is needed. We aimed to validate a model for screening so that only high-risk individuals receive the clinical examination.

METHODS: A community-based case-control study $(n=1029)$ in rural Sri Lanka assessed risk factors and markers for oral potentially malignant disorders (OPMD) by administering a questionnaire followed by an oral examination. We then developed a model based on age, socioeconomic status and habits of betel-quid chewing, alcohol drinking and tobacco smoking, with weightings based on odds ratios from the multiple logistic regression. A total, single score was calculated per individual. Standard receiver-operator characteristic curves were plotted for the total score and presence of OPMD. The model was validated on a new sample of 410 subjects in a different community.

RESULTS: A score of 12.0 produced optimal sensitivity (95.5\%), specificity (75.9\%), false-positive rate (24.0\%), false-negative rate (4.5\%), positive predictive value (35.9\%) and negative predictive value (99.2\%).

CONCLUSION: This model is suitable for detection of OPMD and oral cancer in high-risk communities, for example, in Asia, the Pacific and the global diaspora therefrom. A combined risk-factor score of 12.0 was optimal for participation in oral cancer/OPMD screening in Sri Lanka. The model, or local adaptations, should have wide applicability.

British Journal of Cancer (2010) I 03, 303-309. doi:I0.1038/sj.bjc.6605778 www.bjcancer.com

Published online 13 July 2010

(c) 2010 Cancer Research UK
\end{abstract}

Keywords: oral potentially malignant disorders; oral cancer; risk factors; screening; socioeconomic status; Sri Lanka

Oral and pharyngeal cancer is the sixth most common cancer in the world, with an annual global estimated incidence of about 275000 for oral and 130300 for pharyngeal cancers in the year 2002, excluding salivary neoplasms, malignant neoplasms of the nasopharynx and of the pyriform sinus - two-thirds of these occur in developing countries (Parkin et al, 2005). A particularly high incidence is observed in the Indian sub-continent, which accounts for one-third of the world's burden of oral cancer (Parkin et al, 2001). Rates are also high in Melanesia and other Pacific islands, in parts of Southeast (SE) Asia (Parkin et al, 2001), in Taiwan and some provinces of mainland China where areca nut and/or betel-quid chewing habits are common (Jeng et al, 2001; Gupta and Warnakulasuriya, 2002; Zhang and Reichart, 2007). The incidence of cancer of the oral cavity and oropharynx (excluding salivary neoplasms) in Sri Lanka, standardised to the world population, in the year 2005, was 14.1 and 3.8 per 100000 in males and females, respectively (National Cancer Control Programme Sri Lanka, 2009).

\footnotetext{
*Correspondence: Dr HK Amarasinghe;

E-mail: hemanthaamarasinghe@yahoo.com

Received 22 March 20I0; revised 3 June 2010; accepted II June 2010; published online 13 July 2010
}

Oral cancer is often preceded by so-called 'premalignant lesions' or 'potentially malignant lesions and conditions'. A recent workshop conducted by the WHO Collaborating Centre for Oral Cancer and Precancer in London has recommended the term oral potentially malignant disorders (OPMD) (Warnakulasuriya et al, 2007). The global prevalence of OPMD is reported to be between 1 and $5 \%$ (Napier and Speight, 2008). A high prevalence of OPMD is reported from South and East Asia with male preponderance (Chung et al, 2005; Ariyawardana et al, 2007), and with malignant transformation rates of over $2 \%$ per year (Napier and Speight, 2008). According to the Sri Lankan National Oral Health Survey 2002/2003, it is estimated that more than 284000 people are alive with OPMD, a prevalence of 3.4\% (Ministry of Health Sri Lanka, 2009).

The nature and prevalence of risk factors for OPMDs differ by country and region. In the developing world, tobacco and areca nut used either singly or in various combinations of 'betel quid' or 'pan' (Chung et al, 2005; Ariyawardana et al, 2007), account for the vast majority of the most common OPMD, leukoplakia. We have recently shown that, in Sri Lanka, the population attributable risks for OPMD of daily betel-quid chewing and of regular consumption of alcohol can be estimated at 84 and 25\%, respectively (Amarasinghe et al, 2010). A diet poor in antioxidant vitamins 
and trace elements also constitutes a significant risk factor for oral cancer (Maher et al, 1997; Nagao et al, 2000; Warnakulasuriya, 2009a). Further, recent meta-analyses of the available literature have shown that socioeconomic status (SES) can be considered a significant risk marker for oral cancer, presumably as a surrogate for poor diet, and for heavier use of areca, tobacco and perhaps alcohol (Conway et al, 2008; Warnakulasuriya, 2009b).

In South and Southeast Asia, screening for OPMD has been carried out at various levels and in different settings, ranging from whole communities, targeted to high-risk groups and opportunistically in clinical environments (Warnakulasuriya et al, 1984; Sankaranarayanan et al, 2005; Amarasinghe, 2007). Of these, screening a whole community can be achieved effectively and economically by using an existing health workforce, especially those based within the community itself. A study conducted by one of us in Sri Lanka in the early 1980's using Primary Health Care (PHC) staff in detection of OPMD and oral cancer reported a sensitivity of $89 \%$ (Warnakulasuriya et al, 1984). As a result, this approach has been included in the National Health Policy of Sri Lanka since 1990 (Ministry of Health Sri Lanka, 1990). In spite of this, no sustainable screening programmes have been implemented and Sri Lankan hospitals are reporting an increased proportion of patients with oral cancer, presenting with advanced, often incurable, disease. This is most unfortunate in light of the evidence from the extensive Trivandrum Oral Cancer Screening study, which shows that deaths can be prevented in high-risk communities by such programmes (Sankaranarayanan et al, 2005).

We have determined that the main obstacles to effective oral cancer screening over the intervening three decades include: the lack of adequate guidelines for PHC staff, particularly concerning which individuals should be examined; an excessive workload, including their duties with mothers and babies, and with immunisation programmes; devolution of all vertical preventive programmes to the provincial level; and lack of quality continuing education and assessment systems for health workers (Amarasinghe, 2007). As a possible solution to these problems, we have developed a model designed to identify, in advance, individuals at high risk for oral cancer and for OPMD who can then be targeted for oral examination and for focused preventive measures. Our approach is consonant with the Crete Declaration on Oral Cancer Prevention (Peterson, 2005).

\section{MATERIALS AND METHODS}

This study was carried out in two phases with ethical approval from the ethics review committee, Faculty of Medicine, University of Colombo. Phase 1 consisted of a community-based casecontrol study in the Sabaragamuwa province of rural Sri Lanka. This is located between the western and central provinces and has two districts, Ratnapura and Kegalle. The population characteristics, ethnic mix and socioeconomic diversity is described in detail elsewhere (Amarasinghe, 2008). One administrative area defined by the Medical Officer of Health $(\mathrm{MOH})$ for the district was randomly selected from each district. Ratnapura district has $15 \mathrm{MOH}$ areas, whereas Kegalle district has 10 (Ministry of Health Sri Lanka, 2003). The selected MOH areas contained 42 public health midwife (PHM) areas covering villages and tea and rubber estates - these are considered as cluster units. Of these, 14 clusters were selected using the probabilities proportionate to size sampling technique, with deliberate oversampling of the estate sector. Using a house-to-house method, a total of 1029 willing subjects over 30 years of age were recruited over a 1-year period starting from November 2006.

With the informed signed consent of subjects, trained PHMs administered questionnaires designed to gather sociodemographic and lifestyle information, including details of betel-quid chewing, smoking and consumption of alcohol. Habits were defined in terms of never, ever, past, occasional and daily, as that used in several states in the eastern USA (Morse et al, 2007). Information on occupation and education was amalgamated to represent the SES of the subjects. Anthropometric measurements of height and weight were obtained to calculate body mass index (BMI). Details of diet were obtained, from which the protective effects of fruit and vegetables are reported elsewhere (Amarasinghe, 2008).

A visual oral soft tissue examination was carried out on each subject by the senior author (HKA) for identification of OPMD and any other abnormalities using mouth mirrors both to reflect light and the soft tissues and to examine inaccessible areas of the mouth. The examiner was blinded to the risk-factor status. The diagnostic criteria for the detection of OPMD, namely, leukoplakia, erythroplakia, oral submucous fibrosis (OSF) and lichen planus, were based on the recommendations of the WHO (Axell and Rundquist, 1987; Warnakulasuriya et al, 2007). Other oral mucosal abnormalities were defined according to the workshop held in Kuala Lumpur, Malaysia in 1996 (Zain et al, 1999). HKA has extensive experience of such examinations, and calibrations carried out in 2006 showed high- $\kappa$ agreements with a number of studies carried out by other experienced oral surgeons and oral medicine practitioners (Amarasinghe, 2008).

Subjects with OPMD were taken as cases and those free of both OPMD and any other oral mucosal disease as controls. (Chewers mucosa, quid-induced lichenoid reactions, smoker's keratosis, denture stomatitis, angular cheilitis and oral manifestations of anaemia were considered as other oral mucosal diseases.)

\section{Statistical methods for Phase 1}

Questionnaire and clinical data were recorded on paper, then entered into and analysed by the Statistical Package for Social Sciences (SPSS) Version 17 software package (SPSS inc., IBM, Chicago, IL, USA). Correspondence analysis was used to combine information on occupation and education to represent the SES of the subjects. This was the average of the two scores per individual. The range of scores obtained was then divided into terciles and each individual was placed within one of these bands (Zurriaga et al, 2004). Relationships between two categorical variables were tested by $\chi^{2}$-test. A multivariate logistic regression analysis, including in the model only variables that were statistically significant in the univariate analysis, was used to obtain effect estimates of the potential risk factors on OPMD (Table 1).

Factors to be included into the risk-factor model were based on the results of Phase 1. The risk indicators of age and SES, and the risk factors of betel-quid chewing, tobacco smoking and heavy alcohol drinking were selected. Gradients for each factor were given a score derived from the adjusted odds ratios (ORs) obtained, and were rounded to the nearest whole number to simplify the task for field workers.

A receiver-operator characteristics (ROC) curve was plotted against sensitivity and false-positive rate (FPR) to produce a cutoff point for presence of OPMD, excluding lichen planus. The main reason for excluding subjects with lichen planus in the OPMD group is because the aetiology of this condition, although still poorly understood, is not related to the risk factors included in our risk-factor model (Sugerman et al, 2002; van der Meij et al, 2003; Lodi et al, 2005; Ismail et al, 2007). Because individuals with oral lichen planus and other mucosal diseases are present in the test community, and will be present in other communities to which our risk-factor model might be applied, we have also analysed the data with these disorders included.

The area under the curve (AUC) represents the probability that a random test result will be ranked correctly, as to disease state. 


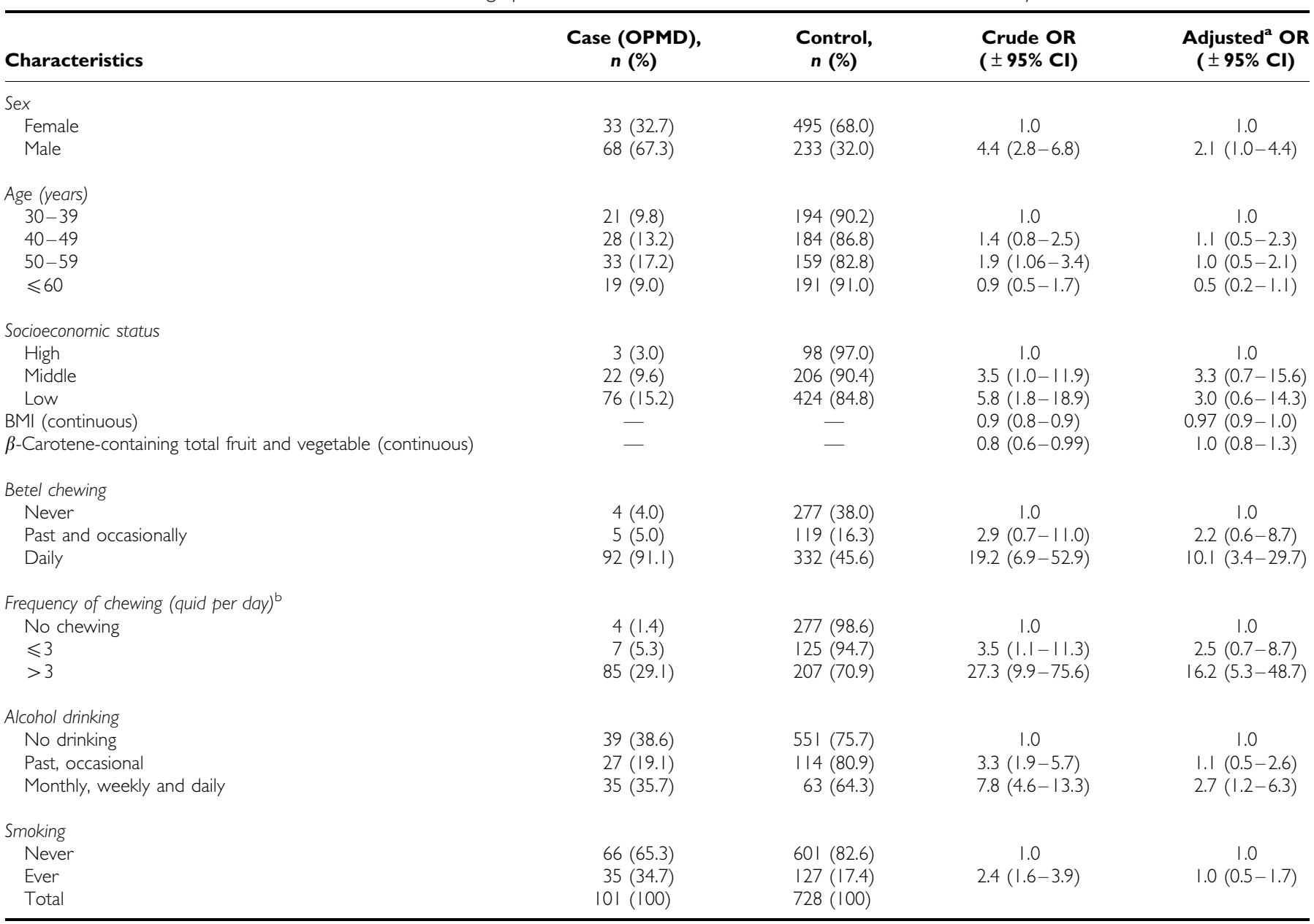

Abbreviations: $\mathrm{BMI}=$ body mass index; $\mathrm{Cl}=$ confidence interval; OPMD = oral potentially malignant disorder; $\mathrm{OR}=$ odds ratio. ${ }^{\mathrm{a}} \mathrm{OR}$ adjusted for sex, age, socioeconomic status,

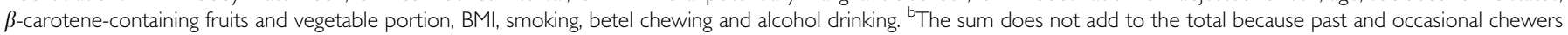
were excluded from the analysis.

Theoretically, a diagonal line would be a score predicting not better than a random guess. Thus, if the AUC is 0.5 or less, the test has no value. The closer the area approaches 1.0, the greater the significance and utility of the test.

In Phase 2, the validation study was carried out in selected PHM areas of the Maharagama MOH, within the suburban population of the Colombo district, and in a rural population in selected PHM areas of the Bulathkohupitiya $\mathrm{MOH}$ area in the Kegalle district of Sri Lanka, over a 4-month period from November 2008. These two $\mathrm{MOH}$ areas were selected because of their ready accessibility, and the existence of on-going oral cancer control activities. The responsible $\mathrm{PHM}$ and a team of up to 10 volunteers advertised, during house-to-house visits, the opportunity for an oral/dental examination and especially encouraged betel and/or tobacco users to attend. Interviews and examinations were conducted locally, the latter using portable dental chairs and headlights. A total of 410 subjects over 15 years of age were recruited.

\section{Statistical methods for Phase 2}

Data were entered and analysed by SPSS as in Phase 1. Each subject was given a score according to the risk-factor model described earlier, and this was compared with the results of the screening examination. The ROC curves were again plotted.

\section{RESULTS}

\section{Phase 1}

A total of 101 cases of previously undiagnosed OPMD were detected by screening. In addition, there were four cases of oral cancer, one newly diagnosed and three under treatment and one case of treated leukoplakia; these were excluded from the analysis. A total of 195 subjects had another oral mucosal disease as defined earlier. There were 728 subjects without any mucosal abnormalities and these were designated as controls for the case-control analysis. When weighted for age and place of residence - namely, village or estate - this represents a prevalence of $11.3 \%$ for OPMD (Amarasinghe et al, 2010).

The risk of occurrence of an OPMD and its statistical significance, according to the logistic regression analysis, is shown in Table 1. Crude ORs were significant for the risk indicators of sex, age and SES, and for the risk factors of betel-quid chewing, smoking, BMI, consumption of alcohol and for intake of $\beta$-carotene containing fruits and vegetables. Betel-quid chewing and consumption of alcohol are the only statistically significant characteristics remaining after controlling for the other factors. After controlling for all other variables, the adjusted OR for daily chewers was 10.1 (95\% CI: 3.4-29.7), with a strong dose-response relation. When considering the consumption of alcohol and risk of OPMD, the adjusted OR for weekly drinkers was 2.7 (95\% CI: 1.2-6.3). 


\section{Derivation of the risk factor model}

Among the lifestyle risk factors, the most critical variables that differentiate disease from non-disease are betel-quid chewing and alcohol use (Table 1). The scores for these were, as described above, derived from the adjusted ORs obtained in Phase 1. Thus, for betel quid, non-chewers scored zero for this parameter, those chewing less than three quids per day scored 2.5 and those chewing three or more quids per day scored 16 (Table 2). Similarly for alcohol use, non-drinkers scored zero, past/occasional drinkers 1 and daily/weekly drinkers 3. Non-smokers were given a score of zero and ever-smokers a score of one. The SES scores were dichotomised to zero for high and 3 for middle and low. For the latter two risk factors/indicators, these values were also taken from the adjusted OR's even though these were not statistically significant, because they have clear importance in many published studies. The weighting for age was established from the literature (Fisher et al, 2005) and from our unpublished data because Phase 1

Table 2 Risk factor model

\begin{tabular}{lc}
\hline Characteristics & Risk score \\
\hline Age (years) & 0 \\
$15-30$ & 3 \\
$>31$ & \\
Socioeconomic status & 0 \\
High & 3 \\
Middle and low & \\
Betel-quid chewing (number of quid per day) & 0 \\
Never & 2.5 \\
$0-3$ & 16 \\
$>3$ & \\
Alcohol drinking & 0 \\
Never & 1 \\
Past, occasional & 3 \\
Daily or at least weekly & \\
Smoking & \\
Never & 0 \\
Ever & 1 \\
\hline
\end{tabular}

did not contain subjects below 30 years of age. The aim was to create a single score that can be easily calculated by adding weighted scores for each factor for each subject. Such scoring systems are currently gaining increasing utility in other health fields, such as cardiology and anaesthesiology (Eberhart et al, 2004; Pepe et al, 2004; Sullivan et al, 2004).

\section{Risk score analysis}

Using all the risk factors and indicators in the model, a ROC curve was plotted for total score assigned to each subject and the presence of OPMD, excluding lichen planus (Figure 1A). A cutoff value of 12 yields maximum length to the diagonal line with an AUC of 0.84 (95\% CI: $0.81-0.87$ ), a sensitivity of $93.7 \%$, a specificity of $67.7 \%$, a FPR of $32.3 \%$, a false-negative rate (FNR) of $6.3 \%$, a positive predictive value (PPV) of $27.5 \%$ and a negative predictive value (NPV) of $98.8 \%$ (Table 3 ).

When all oral mucosal diseases are plotted against the full range of risk indicators and risk factors (Figure 1B), the same optimal cutoff point of 12 emerges, with an AUC of 0.78 (95\% CI: $0.75-0.81$ ), a sensitivity of $81.1 \%$, a specificity of $67.7 \%$, a FPR of $32.3 \%$, a FNR of $18.9 \%$, a PPV of $50.9 \%$ and a NPV of $89.6 \%$ (Table 4).

\section{Phase 2}

Initially 410 subjects were recruited and 3 subjects who had incomplete data were excluded from the analysis. In total, 95 oral mucosal disorders were detected amongst the 407 selected individuals: leukoplakia (34), OSF (10), lichen planus (2) and 49

Table 3 Frequency distribution of cases and controls, applying a 12 cutoff point for the combined score and the presence of OPMD, excluding lichen planus in Phase I study

\begin{tabular}{lccc}
\hline Cutoff value I2 & Cases & Control & Total \\
\hline Disease - above 12 & 89 & 235 & 324 \\
No disease $-<12$ & 6 & 493 & 499 \\
Total & 95 & 728 & 823 \\
\hline
\end{tabular}

Abbreviation: OPMD = oral potentially malignant disorder

ROC curves
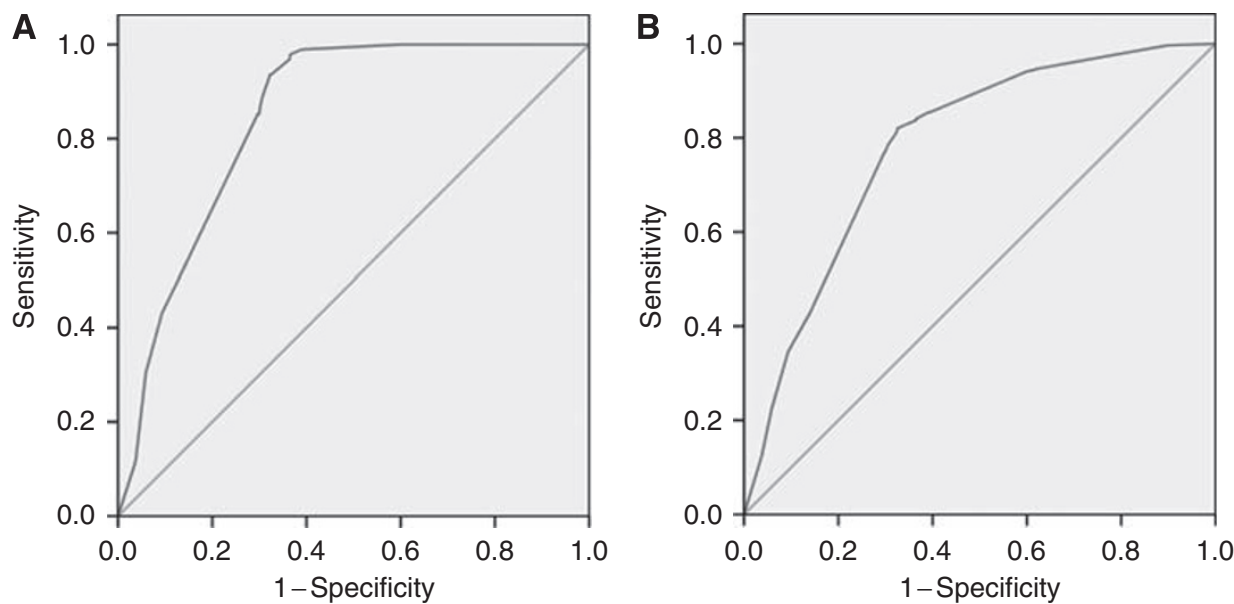

Figure I (A) The ROC curve for risk-factor model score and the presence of OPMD (except lichen planus) in Phase I study. (B) The ROC curve for risk-factor model score and the presence of all oral mucosal disorders in Phase I study. 
with other oral mucosal abnormalities. Using all the risk factors and indicators in the risk-factor model, a ROC curve was plotted for total score assigned to each subject and the same subject's disease status as defined earlier. For OPMD excluding lichen planus (Figure 2A), a cutoff score of 12 yields maximum length to the diagonal line with an AUC of 0.87 (95\% CI: $0.83-0.91)$, a sensitivity of $95.5 \%$, a specificity of $75.9 \%$, a FPR of $24.0 \%$, a FNR of $4.5 \%$, a PPV of $35.9 \%$ and a NPV of $99.2 \%$ (Table 5 ).

When all mucosal diseases are plotted against the full range of risk indicators and risk factors (Figure 2B), the same optimal cutoff score of 12 emerges, with an AUC of 0.80 (95\% CI: $0.75-0.85$ ), sensitivity of $85.3 \%$, a specificity of $75.9 \%$, a FPR of $24.0 \%$, a FNR of $14.7 \%$, a PPV of $51.9 \%$ and a NPV of $94.4 \%$ (Table 6 ).

\section{DISCUSSION}

In Sri Lanka, there is a need for developing a new strategy for the early detection of oral cancer, owing to the high morbidity and mortality associated with the current late presentation of cases (National Cancer Control Programme Sri Lanka, 2009). Moreover, recent studies have shown a dramatic increase in OPMD (Ariyawardana et al, 2007; Amarasinghe et al, 2010). In spite of the fact that oral cancer screening programmes are Government policy, there is low coverage and limited enthusiasm for oral screening amongst PHC staff (Amarasinghe, 2007). This risk-factor model has therefore been developed as a simpler and more costeffective approach to screen high-risk people from any community with a similar risk profile as this study setting, to enable early detection of cases.

In principle, for a screening test to be considered worthwhile, the factors included in any risk-factor model need to be strongly

Table 4 Frequency distribution of cases and controls, applying a 12 cutoff point for the combined score and the presence of all oral mucosal diseases in Phase I study

\begin{tabular}{lrcr}
\hline Cutoff value I & Cases & Control & Total \\
\hline Disease - above 12 & 244 & 235 & 479 \\
No disease $-<12$ & 57 & 493 & 550 \\
Total & 301 & 728 & 1029 \\
\hline
\end{tabular}

associated with the disease(s) of concern, with some variation in exposure to these within the population (Wald et al, 1999). In our Phase 1 case-control study, smoking was not strongly associated with disease, but it was included in the model because of the known importance of this as a risk for upper aerodigestive tract malignancy in many populations (Chung et al, 2005; Ariyawardana et al, 2007; Morse et al, 2007). On the other hand, gender was not retained in the model. This is because, although male gender had an OR of 2.1 in our Phase 1 case-control study, this was not statistically significant (95\% CI: $1.0-4.4)$, and largely reflects higher exposure to combined habits in men. In other populations, women can be the heaviest users of tobacco and betel quid, such as in Malaysian Tamils (Ministry of Health Malaysia, 2003). In the United Kingdom, for example, the male-to-female ratio for the incidence of oral cancer decreased between 1990 and 1999 (Conway et al, 2006), and this was attributed to a reduction in the ratio of male-to-female smokers. We cannot exclude a genetic reason for differences between the sexes, such as has been suggested in relation to oestrogen levels in a Hungarian population

Table 5 Frequency distribution of cases and controls, applying a 12 cutoff point for the combined score and the presence of OPMD, excluding lichen planus in Phase 2 study

\begin{tabular}{lccc}
\hline Cutoff value 12 & Cases & Control & Total \\
\hline Disease - above 12 & 42 & 75 & 117 \\
No disease $-<12$ & 2 & 237 & 239 \\
Total & 44 & 312 & 356 \\
\hline
\end{tabular}

Abbreviation: OPMD = oral potentially malignant disorder.

Table 6 Frequency distribution of cases and controls, applying a 12 cutoff point for the combined score and the presence of all oral mucosal disorders in Phase 2 study

\begin{tabular}{lccc}
\hline Cutoff value I2 & Cases & Control & Total \\
\hline Disease - above 12 & 81 & 75 & 156 \\
No disease $-<12$ & 14 & 237 & 251 \\
Total & 95 & 312 & 407 \\
\hline
\end{tabular}

ROC curves
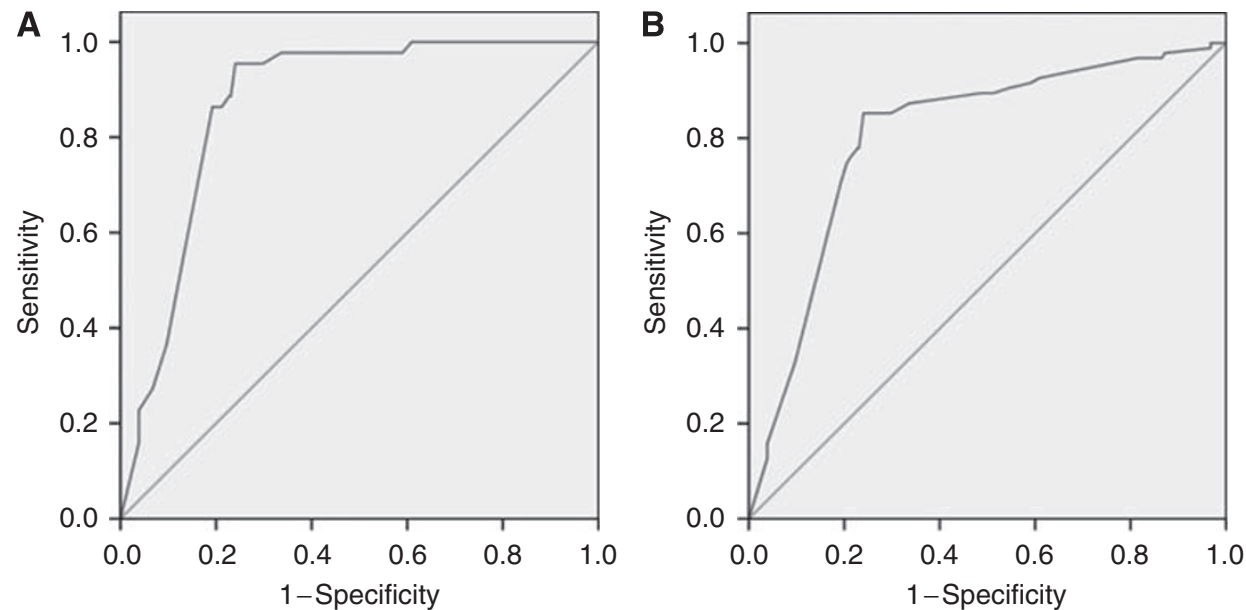

Figure 2 (A) The ROC curve for risk-factor model score and the presence of OPMD (except lichen planus) in Phase 2 validation study. (B) The ROC curve for risk-factor model score and the presence of all oral mucosal disorders in Phase 2 validation study. 
Table 7 Sensitivity and specificity of proposed model for screening compared with test outcomes for other major cancers

\begin{tabular}{lcc}
\hline Disease and test & $\begin{array}{c}\text { Sensitivity } \\
\text { (\%) }\end{array}$ & $\begin{array}{c}\text { Specificity } \\
\text { (\%) }\end{array}$ \\
\hline OPMD using risk-factor model - Phase 1 & 93.7 & 67.7 \\
OPMD using risk-factor model - Phase 2 & 95.5 & 75.9 \\
Breast cancer screening using mammography & $75-90$ & $90-95$ \\
Cervical cancer screening using PAP test & $50-85$ & $95-99$ \\
Colorectal cancer screening with faecal occult & $66-85$ & $95-97$ \\
blood test & & \\
\hline
\end{tabular}

Abbreviation: OPMD = oral potentially malignant disorder.

(Suba, 2007). On balance, we argue that it is better to quantify the risk factors themselves, rather than to confound the risk-factor model with an additional score for sex.

The results show good criterion validity, with outcomes as good as or better than many other cancer screening tests in use today (Table 7), for example, mammography and breast cancer (Ferrini et al, 1996; Taplin et al, 2008), 'PAP smears' and cervical cancer (van der Graaf et al, 1987; De Vuyst et al, 2005; Mayrand et al, 2007) and faecal occult blood and bowel cancer (Rennert et al, 2001; Strul and Arber, 2002; Castiglione and Zappa, 2003).

A recent study from Taiwan has generated ROC curves for individual risk factors with similar results (Yang et al, 2010). However, in the present work, we have combined scores for all risk factors, as this is likely to be more sensitive. The combination is additive because our data from Phase 1 revealed the synergistic effects of betel-quid chewing and alcohol use to be additive. Should subsequent studies in Sri Lanka, especially if they can contribute to a large meta-analysis, reveal multiplicative or intermediary effects, this model can be readily modified. If there are differences in another country or region, similar appropriate modifications can be made.

With a cutoff score of 12 the sensitivity, specificity and NPV of our test for the detection of OPMD is highly satisfactory. Individuals with a score at or above this should be called for a screening examination of the mouth conducted by a trained professional.

The present results show a similar sensitivity - better than $89 \%$ - to that obtained in the pioneering work performed in the 1980 's in the central province of Sri Lanka, in which PHC staff conducted oral examinations for the early detection of oral cancer (Warnakulasuriya et al, 1984). The current FPR is, however, high, which is not a major concern, as these individuals are at high life-time risk and require habit intervention.

A strategy based on our risk-factor model should be simple to implement in terms of logistics, time and money, especially compared with existing systems, which require physical examination of whole populations. Grass-root level health volunteers or estate welfare officers can be used to select high-risk people for subsequent oral screening by trained personnel. The PHC staff can be used to supervise a programme and to arrange referral to the nearest dental clinic or organise visits by mobile clinics. The main advantage of this approach is that professional clinicians are needed only when individuals at high risk reach the clinical setting.

This approach can be integrated within the existing PHC strategies of the Sri Lankan Government or be adapted to a social marketing strategy. The latter approach has been used successfully by the anti-leprosy campaign in Sri Lanka to detect early cases of this infectious disease (Ministry of Health Sri Lanka, 2003). With such an approach, it is desirable to have a single clear message delivered consistently through all available media.

It is a common observation with screening tests that detecting those unlikely to have or to develop a disease is easier than detecting those with disease or at high risk; nevertheless, as this is the majority of a given population, the information is valuable and cost-effective (Johnson, 1991). The approach, with minor variations apposite to local lifestyles and culture, is applicable to those many countries with high incidence of oral cancer, and to the diaspora therefrom who carry their risky lifestyles with them.

\section{Limitations of the risk-factor model}

Ethical issues arise from the FPR, because a quarter to one-third of the population will be asked to attend a mouth examination when in fact some may not have the target disease. Anxiety will be generated in subjects and their families when referral for a physical examination is made. This will also have cost implications for the patient and for providers. However, the physical discomfort involved is minimal, and a visit to a clinic provides an opportunity for habit intervention and other health screening.

Inevitably, there will also be false-negative cases, who will miss an opportunity for detection of oral disease if not referred for physical examination. For example, a minor proportion of oral cancers and of OPMD arise from factors other than betel quid, tobacco and heavy alcohol use - perhaps associated with genetic predisposition or infection with human papillomavirus or idiopathic; nevertheless, on a global scale, tobacco and other environmental risk factors vastly outweigh these as causes of the burden of disease (Axell, 1987; Herrero et al, 2003).

Finally, this particular model applies to the communities described herein and cannot be taken to other very different communities, for example, those in the Western world, in which areca nut habits are uncommon. The approach, however, should be widely applicable, using appropriate weightings for smoking, alcohol and other risk factors known for that particular community.

\section{ACKNOWLEDGEMENTS}

We gratefully acknowledge the WHO for funding and the contributions made by Dr Jayasundara Bandara, Dr Udaya Usgodaarachchi, Dr Lakal Dissabandara, Dr (Ms) Irosha Perera, the dental surgeons and staff of the Dental Public Health Unit, Dental Institute, Maharagama, staff of the $\mathrm{MOH}$ Bulathkohupitiya and the participants and their families.

\section{REFERENCES}

Amarasinghe AAHK (2007) Early detection of oral cancer in the estate sector and the urban areas in the Kalutara district of Sri Lanka: a comparison of utilization of health staff and health volunteers. Sri Lanka Dent J 37: $93-98$

Amarasinghe AAHK (2008) The Prevalence of Risk Factors and the Level of Public Awareness of Oral Potentially Malignant Disorders in the Sabaragamuwa Province of Sri Lanka MD thesis, Post Graduate Institute of Medicine, Sri Lanka

Amarasinghe AAHK, Usgodaarachchi US, Johnson NW, Lalloo R, Warnakulasuriya S (2010) Betel-quid chewing with or without tobacco

is a major risk factor for oral potentially malignant disorders in Sri Lanka: a case-control study. Oral Oncol 46(4): 297-301

Ariyawardana A, Sitheeque MA, Ranasinghe AW, Perera I, Tilakaratne WM, Amaratunga EA, Yang YH, Warnakulasuriya S (2007) Prevalence of oral cancer and pre-cancer and associated risk factors among tea estate workers in the central Sri Lanka. J Oral Pathol Med 36: 581-587

Axell T (1987) Occurrence of leukoplakia and some other oral white lesions among 20333 adult Swedish people. Community Dent Oral Epidemiol 15: 46-51

Axell T, Rundquist L (1987) Oral lichen planus - a demographic study. Community Dent Oral Epidemiol 15: 52-56 
Castiglione G, Zappa M (2003) Debate on colorectal cancer screening by faecal occult blood. Ann Oncol 14: 342-344

Chung CH, Yang YH, Wang TY, Shieh TY, Warnakulasuriya S (2005) Oral precancerous disorders associated with areca quid chewing, smoking, and alcohol drinking in southern Taiwan. J Oral Pathol Med 34: 460 - 466

Conway DI, Petticrew M, Marlborough H, Berthiller J, Hashibe M, Macpherson MD (2008) Socioeconomic inequalities and oral cancer risk: a systematic review and meta-analysis of case-control studies. Int J Cancer 122: 2811-2819

Conway DI, Stockton DL, Warnakulasuriya KA, Ogden G, Macpherson LM (2006) Incidence of oral and oropharyngeal cancer in United Kingdom (1990-1999) - recent trends and regional variation. Oral Oncol 42: $586-592$

De Vuyst H, Claeys P, Njiru S, Muchiri L, Steyaert S, De Sutter P, Van Marck E, Bwayo J, Temmerman M (2005) Comparison of PAP smear, visual inspection with acetic acid, human papillomavirus DNA-PCR testing and cervicography. Int J Gynaecol Obstet 89: 120-126

Eberhart LHJ, Geldner G, Kranke P, Morin AM, Schauffelen A, Treiber H, Wulf $\mathrm{H}$ (2004) The development and validation of a risk score to predict the probability of postoperative vomiting in pediatric patients. Anesth Analg 99: $1630-1637$

Ferrini R, Mannino E, Ramsdell E, Hill L (1996) Screening mammography for breast cancer: American College of Preventive Medicine practice policy statement. Available at: http://www.acpm.org/breast.htm. Accessed on 15 March 2010

Fisher MA, Bouquot JE, Shelton BJ (2005) Assessment of risk factors for oral leukoplakia in West Virginia. Community Dent Oral Epidemiol 33: 45-52

Gupta PC, Warnakulasuriya S (2002) Global epidemiology of areca nut usage. Addict Biol 7: 77-83

Herrero R, Castellsague X, Pawlita M, Lissowska J, Kee F, Balaram P, Rajkumar T, Sridhar H, Rose B, Pintos J, Fernandez L, Idris A, Sanchez MJ, Nieto A, Talamini R, Tavani A, Bosch FX, Reidel U, Snijders PJ, Meijer CJ, Viscidi R, Munoz N, Franceschi S (2003) Human papillomavirus and oral cancer: the International Agency for Research on Cancer multicenter study. J Natl Cancer Inst 95: 1772 - 1783

Ismail SB, Kumar SK, Zain RB (2007) Oral lichen planus and lichenoid reactions: etiopathogenesis, diagnosis, management and malignant transformation. J Oral Sci 49: 89-106

Jeng JH, Chang MC, Hahn LJ (2001) Role of areca nut in betel quidassociated chemical carcinogenesis: current awareness and future perspectives. Oral Oncol 37: 477-492

Johnson NW (1991) New knowledge concerning oral cancer and research priorities. Chapter 18. In: Oral Cancer-Detection of Patients and Lesions at Risk, Cambridge University Press: Cambridge; New York; Melbourne. pp 389-393

Lodi G, Scully C, Carrozzo M, Griffiths M, Sugerman PB, Thongprasom K (2005) Current controversies in oral lichen planus: report of an international consensus meeting. Part 2. Clinical management and malignant transformation. Oral Surg Oral Med Oral Pathol Oral Radiol Endod 100: $164-178$

Maher R, Aga P, Johnson NW, Sankaranarayanan R, Warnakulasuriya S (1997) Evaluation of multiple micronutrient supplementation in the management of oral submucous fibrosis in Karachi, Pakistan. Nutr Cancer 27: $41-47$

Mayrand MH, Duarte-Franco E, Rodrigues I, Walter SD, Hanley J, Ferenczy A, Ratnam S, Coutlee F, Franco EL (2007) Human papillomavirus DNA versus Papanicolaou screening tests for cervical cancer. $N$ Engl J Med 357: $1579-1588$

Ministry of Health Malaysia (2003) Second report of the National Cancer Registry, Cancer Incidence in Malaysia 2003. Available from http://www.radiologymalaysia.org/Archive/NCR/2ndNCR.pdf. Accessed on 09 December 2009

Ministry of Health Sri Lanka (1990) National Health Policy of Sri Lanka. Ministry of Health Sri Lanka: Colombo

Ministry of Health Sri Lanka (2003) Annual Health Bulletin. Ministry of Health Sri Lanka: Colombo. 62-64

Ministry of Health Sri Lanka (2009) National Oral Health Survey, Sri Lanka (2002/2003). Ministry of Health Sri Lanka: Colombo, 3rd publication

Morse DE, Poster WJ, Cleveland D, Cohen D, Mohit-Tabatabai M, Kosis DL, Eisenberg E (2007) Smoking and drinking in relation to oral cancer and oral epithelial dysplasia. Cancer Causes Control 18: 919-929
Nagao T, Ikeda N, Warnakulasuriya S, Fukano H, Yuasa H, Yano M, Miyazaki H, Ito Y (2000) Serum antioxidant micronutrients and the risk of oral leukoplakia among Japanese. Oral Oncol 36: $466-470$

Napier SS, Speight PM (2008) Natural history of potentially malignant oral lesions and conditions: an overview of the literature. J Oral Pathol Med 37: $1-10$

National Cancer Control Programme Sri Lanka (2009) Cancer Incidence Data: Sri Lanka Year 2001-2005. NCCP: Colombo 7th Publication

Parkin DM, Bray F, Ferlay J, Pisani P (2001) Estimating the world cancer burden: Globocan 2000. Int J Cancer 94: 153-156

Parkin DM, Bray F, Ferlay J, Pisani P (2005) Global cancer statistics, 2002. CA Cancer J Clin 55: 74-108

Pepe MS, Janes H, Longton G, Leisenring W, Newcomb P (2004) Limitation of the odds ratio in gauging the performance of a diagnostic, prognostic, or screening marker. Am J Epidemiol 159: $882-890$

Peterson PE (2005) Strengthening the prevention of oral cancer: the WHO perspective. Community Dent Oral Epidemiol 33: 397-399

Rennert G, Rennert HS, Miron E, Peterburg Y (2001) Population colorectal cancer screening with fecal occult blood test. Cancer Epidemiol Biomarkers Prev 10: $1165-1168$

Sankaranarayanan R, Ramadas K, Thomas G, Muwonge R, Thara S, Mathew B, Rajan B (2005) Effect of screening on oral cancer mortality in Kerala, India: a cluster-randomised controlled trial. Lancet 365: 1927 - 1933

Strul H, Arber N (2002) Fecal occult blood test for colorectal cancer screening. Ann Oncol 13: 51-56

Suba Z (2007) Gender-related hormonal risk factors for oral cancer. Pathol Oncol Res 13: 195-202

Sugerman PB, Savage NW, Walsh LJ, Zhao ZZ, Zhou XJ, Khan A, Seymour GJ, Bigby M (2002) The pathogenesis of oral lichen planus. Crit Rev Oral Biol Med 13: $350-365$

Sullivan ML, Massaro JM, D'Agostino Sr RB (2004) Presentation of multivariate data for clinical use:The Framingham study risk score functions. Stat Med 23: $1631-1660$

Taplin S, Abraham L, Barlow WE, Fenton JJ, Berns EA, Carney PA, Cutter GR, Sickles EA, Carl D, Elmore JG (2008) Mammography facility characteristics associated with interpretive accuracy of screening mammography. J Natl Cancer Inst 100: 876 - 887

van der Graaf Y, Vooijs GP, Gaillard HL, Go DM (1987) Screening errors in cervical cytologic screening. Acta Cytol 31: 434-438

van der Meij EH, Schepman KP, van der Waal I (2003) The possible premalignant character of oral lichen planus and oral lichenoid lesions: a prospective study. Oral Surg Oral Med Oral Pathol Oral Radiol Endod 96: $164-171$

Wald NJ, Hackshaw AK, Frost CD (1999) When can a risk factor be used as a worthwhile screening test? $\mathrm{Br}$ Med J 19: $1562-1565$

Warnakulasuriya KA, Ekanayake AN, Sivayoham S, Stjernsward J, Pindborg JJ, Sobin LH, Perera KS (1984) Utilization of primary health care workers for early detection of oral cancer and precancer cases in Sri Lanka. Bull World Health Organ 62: 243-250

Warnakulasuriya S (2009a) Food, nutrition and oral cancer. In: Michael Wilson (ed) Food constituents and oral health. Woodhead Publishing Limited: Oxford. pp 273-295

Warnakulasuriya S (2009b) Significant oral cancer risk associated with low socioeconomic status. Evid Based Dent 10: 4-5

Warnakulasuriya S, Johnson NW, van der Waal I (2007) Nomenclature and classification of potentially malignant disorders of the oral mucosa. J Oral Pathol Med 36: 575-580

Yang YH, Ho PS, Lu HM, Huang IY, Chen CH (2010) Comparing doseresponse measurements of oral habits on oral leukoplakia and oral submucous fibrosis from a community screening program. J Oral Pathol Med 39(14): 306-312

Zain RB, Ikeda N, Gupta PC, Warnakulasuriya S, van Wyk CW, Shrestha P, Axell T (1999) Oral mucosal lesions associated with betel quid, areca nut and tobacco chewing habits: consensus from a workshop held in Kuala Lumpur, Malaysia, November 25-27, 1996. J Oral Pathol Med 28: $1-4$

Zhang X, Reichart PA (2007) A review of betel quid chewing, oral cancer and precancer in Mainland China. Oral Oncol 43: 424-430

Zurriaga O, Martinez-Beneito MA, Abellan JJ, Carda C (2004) Assessing the social class of children from parental information to study possible social inequalities in health outcomes. Ann Epidemiol 14: 378-384 